\title{
An augmented Lagrangian SQP method for solving some special class of nonlinear semi-definite programming problems
}

\author{
EL-SAYED M.E. MOSTAFA \\ Department of Mathematics, Faculty of Science, Alexandria Univ., Alexandria, Egypt \\ E-mail: emostafa99@yahoo.com
}

\begin{abstract}
In this paper, we consider a special class of nonlinear semi-definite programming problems that represents the fixed order $\mathcal{H}_{2} / \mathcal{H}_{\infty}$ synthesis problem. An augmented Lagrangian sequential quadratic programming method combined with a trust region globalization strategy is described, taking advantage of the problem structure and using inexact computations. Some numerical examples that illustrate the performance of the method are given.
\end{abstract}

Mathematical subject classification: 49N35, 49N10, 93D52, 93D22, $65 \mathrm{~K} 05$.

Key words: semi-definite programming, linear quadratic control, nonlinear programming, trust region methods.

\section{Introduction}

In this paper, the following nonlinear semi-definite programming (NSDP) problem is considered:

$$
(\mathrm{NSDP}): \begin{cases}\min _{X, V} & J(X) \\ \text { s.t. } & H(X)=0, Y(X, V) \preceq 0, V \succ 0,\end{cases}
$$

where $J: \mathbb{R}^{p \times r} \times S^{n} \rightarrow \mathbb{R}, H: \mathbb{R}^{p \times r} \times S^{n} \rightarrow S^{n}, Y: \mathbb{R}^{p \times r} \times S^{n} \times S^{n} \rightarrow S^{n}$ are assumed to be sufficiently smooth matrix functions and $S^{n}$ denotes the set of real symmetric $n \times n$ matrices. This problem is a nonlinear matrix programming problem and is generally nonconvex. 
The augmented Lagrangian function associated with the equality constraint of (1) is defined as

$$
\mathcal{L}^{\sigma}(X, K)=J(X)+\langle K, H(X)\rangle+\frac{\sigma}{2}\|H(X)\|^{2}, \sigma \geq 0,
$$

where $K \in \mathbb{R}^{n \times n}$ is the associated Lagrange multiplier and $\sigma$ is the penalty parameter.

Several problems in system and control theory can be reduced to some special class of NSDPs (see, e.g., [3], [1], [10], [6], [12]). NSDP formulations of control problems were made popular in the mid of 1990s. There were, however, no computational methods for solving general nonconvex NSDPs. Recently, nonlinear optimization techniques have been employed to solve NSDPs arising in optimal control (see, e.g., [1], [10], [6], [12]).

The main goal of this paper is to propose an augmented Lagrangian sequential quadratic programming (ALSQP) Algorithm that makes use of trust region for finding an approximate solution to (1). ALSQP methods have shown to be quite successful in solving nonlinear programming (NLP) problems. In particular, an ALSQP approach is effective in solving NLP problems, even when the problem is ill-conditioned or the constraints are highly nonlinear. We use trust region strategies to globalize the ALSQP iteration, because they facilitate the use of second derivative information when the problem is nonconvex. The reader is referred, for instance, to the book of Conn, Gould and Toint [4] for a survey of augmented Lagrangian methods and trust region methods.

The difficulties in solving (1) are due to the fact that this problem is a nonlinear and nonconvex matrix programming problem. NSDP formulations of optimal control applications own special structures that are desirably exploited. Having this in mind, we seek in the proposed ALSQP method to combine ideas of SDPapproaches, sequential quadratic programming, and trust region to construct an optimization solver for (1) that exploits the inherent structure of the considered NSDP problem.

This paper is organized as follows. In the next subsection we state the basic assumptions imposed on the problem NSDP. In addition, we discuss the framework of the ALSQP Algorithm. In $\S 2$ we present the formulation of the considered problem. In $\S 3$ we introduce the constrained trust region Algorithm for solving 
the quadratic programming trust region subproblem associated with the problem NSDP. In $\S 4$ we test numerically the performance of the ALSQP method through several test problems from the benchmark collection COMPleib [8].

Notations. For a matrix $M \in \mathbb{R}^{n \times n}$ the notations $M \succ 0, M \succeq 0, M \prec 0$, $M \preceq 0$ denote that $M$ is positive definite, positive semi-definite, negative definite, negative semi-definite, respectively. Sometimes the arguments as in $H(\cdot)$ are omitted, which should be obvious from the context. Throughout the paper, $\|\cdot\|$ denotes the Frobenius norm given by $\|M\|=\sqrt{\langle M, M\rangle}$, where $\langle\cdot, \cdot\rangle$ is the inner product defined by $\left\langle M_{1}, M_{2}\right\rangle=\operatorname{Tr}\left(M_{1}^{T} M_{2}\right)$, and $\operatorname{Tr}(\cdot)$ is the trace operator.

\subsection{Outline of the Algorithm ALSQP and assumptions}

In this subsection, we state the basic assumptions imposed on the problem NSDP. In addition, we describe the framework of the ALSQP Algorithm.

Assumption 1.1. The following basic assumptions are used throughout the paper:

AS1. $J$ and $H$ are twice continuously differentiable in an open neighborhood of the local solution $X_{*}$, and their second derivatives are Lipschitz continuous at $X_{*}$.

AS2. There exist $\left(X_{0}, V_{0}\right) \in \mathcal{F}_{s}$, where

$$
\mathcal{F}_{s}=\left\{(X, V) \in \mathbb{R}^{p \times r} \times S^{n} \times S^{n} \mid Y(X, V) \prec 0, V \succ 0\right\} .
$$

AS3. The second-order sufficient optimality conditions hold at the solution, i.e., there exists a constant $\kappa>0$ satisfying

$$
\begin{aligned}
\mathcal{L}_{X X}^{0}\left(X_{*}, K_{*}\right)(\Delta X, \Delta X) \geq & \kappa_{0}\|\Delta X\|^{2} \quad \forall \quad \Delta X \in \mathbb{R}^{p \times r} \times \mathbb{R}^{n \times n} \\
& \text { such that } H_{X}\left(X_{*}\right) \Delta X=0 .
\end{aligned}
$$

AS4. The mapping $\nabla H\left(X_{*}\right)$ is surjective.

AS5. There exists $\left(X_{*}, V_{*}\right)$ solution of NSDP such that $V_{*} \succ 0, Y\left(X_{*}, V_{*}\right) \prec 0$. 
Note that the surjectivity assumption is the classical regularity assumption of nonlinear programming.

ALSQP methods are iterative. The search directions in these methods are obtained by solving a sequence of quadratic programs (QP). Each QP minimizes at every iteration $k$ a quadratic model of a certain augmented Lagrangian function subject to linearized constraints; in our case the QP takes the form:

$$
(\mathrm{QPTR}):\left\{\begin{array}{cl}
\min _{(\Delta X, \Delta V)} & \mathcal{L}_{X}^{\sigma}\left(X_{k}, K_{k}\right) \Delta X+\frac{1}{2} \mathcal{L}_{X X}^{\sigma}\left(X_{k}, K_{k}\right)(\Delta X, \Delta X) \\
\text { s.t. } & H_{X}\left(X_{k}\right) \Delta X+H\left(X_{k}\right)=0, \quad\|\Delta X\| \leq \delta, \\
& \left(X_{k}+\Delta X, V_{k}+\Delta V\right) \in \mathcal{F}_{s},
\end{array}\right.
$$

where the trust region constraint $\|\Delta X\| \leq \delta$ ( $\delta>0$ is the trust-region radius) is included to avoid possible unboundedness in the QP.

Given the current estimate $\left(X_{k}, V_{k}\right)$ of the solution of the problem NSDP and the Lagrange multiplier $K_{k}$, the following Algorithm explains how to obtain the new iterate $\left(X_{k}+\Delta X, V_{k}+\Delta V\right)$.

Algorithm 1.1. (Algorithm ALSQP for solving the problem NSDP)

a. Choose $\left(X_{0}, V_{0}\right) \in \mathcal{F}_{s}, K_{0} \in \mathbb{R}^{n \times n}$, and $\sigma_{0} \geq 0$. Set $k:=0$ and go to the next step.

b. If the prescribed stopping criterion is reached, then stop; otherwise continue with the next step.

c. At every iteration $k$ compute an approximate solution $(\Delta X, \Delta V)$ to the problem QPTR such that $\left(X_{k}+\Delta X, V_{k}+\Delta V\right) \in \mathcal{F}_{s}$.

d. Set $\left(X_{k+1}, V_{k+1}\right)=\left(X_{k}+\Delta X, V_{k}+\Delta V\right)$, update $K_{k+1}$, set $k=k+1$, and go to step $b$.

Some comments are now in order.

- Obviously, it is not trivial how to obtain an initial $\left(X_{0}, V_{0}\right) \in \mathcal{F}_{s}$ (item $a$ of the ALSQP Algorithm). In $\S 4$, however, we describe a technique for determining $\left(X_{0}, V_{0}\right) \in \mathcal{F}_{s}$ that relies on Lyapunov stability theory. 
- The multiplier $K$ can be updated without extra calculations while taking problem structure into consideration; see the end of $\$ 2$ and the Algorithm 3.2.

The Algorithm ALSQP terminates if the following criterion is satisfied

$$
\left\|\nabla_{X} \mathcal{L}^{\sigma_{k}}\left(X_{k}, K_{k}\right)\right\|+\left\|H\left(X_{k}\right)\right\| \leq \epsilon_{t o l},
$$

where $\epsilon_{t o l}>0$ is the tolerance.

\section{Problem formulation/application}

In optimal control, an important nonlinear and nonconvex application is the problem of designing a static output feedback (SOF) control law that meets a desired performance criterion. A typical instance of an output feedback control system can be stated as follows. Consider a linear time-invariant state space model of order $n_{x}$,

$$
\begin{aligned}
\dot{x}(t) & =A x(t)+B u(t)+B_{1} w(t), \\
y(t) & =C x(t), \\
z(t) & =C_{1} x(t)+D_{1} u(t),
\end{aligned}
$$

where $x \in \mathbb{R}^{n_{x}}, w \in \mathbb{R}^{n_{w}}, u \in \mathbb{R}^{n_{u}}, z \in \mathbb{R}^{n_{z}}$, and $y \in \mathbb{R}^{n_{y}}$ denote the state, the disturbance input, the control input, the regulated output, and the measured output, respectively. Furthermore, $A, B, B_{1}, C, C_{1}$, and $D_{1}$ are given constant matrices of appropriate size.

The static output feedback control law is given by

$$
u(t)=F y(t)
$$

where $F \in \mathbb{R}^{n_{u} \times n_{y}}$ is unknown.

Substituting the control law (7) into our control system, then the closed loop counterpart yields:

$$
\Sigma_{c l}:\left\{\begin{aligned}
\dot{x}(t) & =A(F) x(t)+B(F) w(t), \\
z(t) & =C(F) x(t),
\end{aligned}\right.
$$

where $A(F):=A+B F C, B(F):=B_{1}, C(F):=C_{1}+D_{1} F C$ are the augmented closed loop operators, respectively. 
The fixed order $\mathcal{H}_{2} / \mathcal{H}_{\infty}$ synthesis problem (see, e.g., [2], [5], [7]) is equivalent to the following NSDP problem:

$$
(\mathrm{NSDP} 1):\left\{\begin{aligned}
& \min \quad J(L)=\operatorname{Tr}\left(L B_{1} B_{1}^{T}\right) \\
& \text { s.t. } \quad H(F, L)=A(F)^{T} L+L A(F)+C(F)^{T} C(F) \\
&+\frac{1}{\gamma^{2}} L B_{1} B_{1}^{T} L=0, \\
& Y(F, L, V)=\tilde{A}(\cdot)^{T} V+V \tilde{A}(\cdot) \prec 0, \quad V \succ 0,
\end{aligned}\right.
$$

where

$$
\tilde{A}(\cdot)=\left(A(F)+\frac{1}{\gamma^{2}} B_{1} B_{1}^{T} L\right),
$$

and $\gamma>0$ is a given constant. Clearly, the problem NSDP is a generalization of the problem NSDP1, where $X=(F, L) \in \mathbb{R}^{p \times r} \times S^{n}$.

Observe that, if we assign a large constant value to $\gamma$ in (9), then we obtain the following special case that corresponds to the fixed order $\mathcal{H}_{2}$ synthesis problem (see, e.g., [7], [9], [14]):

$$
(\mathrm{NSDP} 2): \begin{cases}\min \quad & J(L)=\operatorname{Tr}\left(L B_{1} B_{1}^{T}\right) \\ \text { s.t. } \quad & \hat{H}(F, L)=A(F)^{T} L+L A(F)+C(F)^{T} C(F)=0, \\ & \hat{Y}(F, V)=A(F)^{T} V+V A(F) \prec 0, \quad V \succ 0 .\end{cases}
$$

Note, however, that the ALSQP method reduces to solving (10) by simply assigning a large constant value to $\gamma$ in that method.

First and second-order derivatives of the augmented Lagrangian function (2) are obtained in the following Lemma, which will be needed later on to construct the trust region problem.

Lemma 2.1 [10, lemma 2.1]. Let $(F, L, V) \in \mathcal{F}_{s}, K \in \mathbb{R}^{n \times n}$ be given. Then, the function $J$ and the constraint function $H$ are twice continuously differentiable on $\mathcal{F}_{s}$. Furthermore, the first and the second order directional derivatives of 
$\mathcal{L}^{\sigma}(F, L, K)$ are given by

$$
\begin{aligned}
& \mathcal{L}_{F}^{\sigma}(\cdot) \Delta F \equiv\left\langle\Delta F, \nabla_{F} \mathcal{L}^{\sigma}(\cdot)\right\rangle=\left\langle\Delta F, 2 N(F, L)(K+\sigma H(\cdot)) C^{T}\right\rangle, \\
& \mathcal{L}_{L}^{\sigma}(\cdot) \Delta L \equiv\left\langle\Delta L, \nabla_{L} \mathcal{L}^{\sigma}(\cdot)\right\rangle=\langle\Delta L, \tilde{A}(\cdot)(K+\sigma H(\cdot)) \\
&\left.+(K+\sigma H(\cdot)) \tilde{A}(\cdot)^{T}+B_{1} B_{1}^{T}\right\rangle, \\
& \mathcal{L}_{F F}^{\sigma}(\cdot)(\Delta F, \Delta F) \equiv\left\langle\Delta F, \nabla_{F F}^{2} \mathcal{L}^{\sigma}(\cdot) \Delta F\right\rangle, \\
& \mathcal{L}_{F L}^{\sigma}(\cdot)(\Delta F, \Delta L) \equiv\left\langle\Delta F, \nabla_{F L}^{2} \mathcal{L}^{\sigma}(\cdot) \Delta L\right\rangle=\mathcal{L}_{L F}^{\sigma}(\cdot)(\Delta L, \Delta F), \\
& \mathcal{L}_{L L}^{\sigma}(\cdot)(\Delta L, \Delta L) \equiv\left\langle\Delta L, \nabla_{L L}^{2} \mathcal{L}^{\sigma}(\cdot) \Delta L\right\rangle
\end{aligned}
$$

where $K$ is the solution of the adjoint equation,

$$
\begin{aligned}
\nabla_{F F}^{2} \mathcal{L}^{\sigma}(\cdot) \Delta F= & 2 D_{1}^{T} D_{1} \Delta F C(K+\sigma H(\cdot)) C^{T}+2 \sigma N(F, L) N(F, L)^{T} \Delta F C C^{T} \\
& +2 \sigma N(F, L) C^{T} \Delta F^{T} N(F, L) C^{T}, \\
\nabla_{F L}^{2} \mathcal{L}^{\sigma}(\cdot) \Delta L= & 2\left(B^{T} \Delta L(K+\sigma H(\cdot)) C^{T}+2 \sigma N(F, L) H_{L} \Delta L C^{T}\right. \\
\nabla_{L L}^{2} \mathcal{L}^{\sigma}(\cdot) \Delta L= & \sigma\left(\tilde{A}(\cdot) H_{L}(\cdot) \Delta L+H_{L}(\cdot) \Delta L \tilde{A}(\cdot)^{T}\right) \\
& +\frac{2}{\gamma^{2}}\left(B_{1} B_{1}^{T} \Delta L(K+\sigma H(\cdot))\right),
\end{aligned}
$$

and the directional derivatives of $H(F, L)$ with respect to $F$ and $L$ are

$$
\begin{aligned}
& H_{F}(\cdot) \Delta F \quad \equiv\left\langle\Delta F, \nabla_{F} H(\cdot)\right\rangle=C^{T} \Delta F^{T} N(F, L)+N(F, L)^{T} \Delta F C, \\
& H_{L}(\cdot) \Delta L \equiv\left\langle\Delta L, \nabla_{L} H(\cdot)\right\rangle=\Delta L \tilde{A}(\cdot)+\tilde{A}(\cdot)^{T} \Delta L
\end{aligned}
$$

where

$$
\tilde{A}(\cdot)=\left(A(F)+\frac{1}{\gamma^{2}} B_{1} B_{1}^{T} L\right), N(F, L)=\left(B^{T} L+D_{1}^{T} C(F)\right) .
$$

Moreover, the Hessian of the augmented Lagrangian is Lipschitz continuous.

Proof. The differentiability of $J$ and $H$ is straightforward. First and second order directional derivatives of $\mathcal{L}^{\sigma}$ with respect to $F$ and $L$ yield the above equations (See, e.g., [11] for a similar result, but with using the Lagrangian function and not the augmented Lagrangian). 
However, it is also possible to obtain those derivatives of $\mathcal{L}^{\sigma}(X, K)$ from their corresponding counterparts of $\mathcal{L}^{0}(X, K)$ according to the fact:

$$
\begin{aligned}
& \mathcal{L}_{X}^{\sigma}(\cdot, K ; \cdot) \Delta X=\mathcal{L}_{X}^{0}(\cdot, K+\sigma H ; \cdot) \Delta X, \\
& \mathcal{L}_{X X}^{\sigma}(\cdot, K ; \cdot)(\Delta X, \Delta X)=\mathcal{L}_{X X}^{0}(\cdot, K+\sigma H ; \cdot)(\Delta X, \Delta X) .
\end{aligned}
$$

By using the result of Lemma 2.1 the first-order necessary optimality conditions for the problem NSDP1 are:

$$
\begin{aligned}
& \nabla_{F} \mathcal{L}^{\sigma}(\cdot)=\left(B^{T} L+D_{1}^{T} C(F)\right)(K+\sigma H(\cdot)) C^{T}=0, \\
& \nabla_{L} \mathcal{L}^{\sigma}(\cdot)=\tilde{A}(\cdot)(K+\sigma H(\cdot))+(K+\sigma H(\cdot)) \tilde{A}(\cdot)^{T}+B_{1} B_{1}^{T}=0, \\
& H(\cdot)=L \tilde{A}(\cdot)+\tilde{A}(\cdot)^{T} L+C(F)^{T} C(F)=0,
\end{aligned}
$$

where $\tilde{A}(\cdot)=\left(A(F)+\frac{1}{\gamma^{2}} B_{1} B_{1}^{T} L\right)$. For optimal control problems (14) represents the state equation, (13) corresponds to the adjoint equation, and the lefthand side of (12) corresponds to the gradient. It is worth noting that, for $\sigma=0$ the adjoint equation (13) reduces to a Lyapunov equation that can be employed to determine a new Lagrange multiplier estimate in the proposed method.

Observe that, if the penalty parameter $\sigma$ is set to zero in (12)-(14), then the Karush-Kuhn-Tucker (KKT) system for the problem NSDP1 is obtained. On the other hand, if $\gamma \rightarrow+\infty$ in (12)-(14) and $\sigma=0$, the KKT system of the problem NSDP2 is achieved. In this case, $\tilde{A}(F, L) \rightarrow A(F), H(F, L) \rightarrow$ $\hat{H}(F, L)$, and $Y(F, L, V) \rightarrow \hat{Y}(F, V)$.

\section{Constrained trust region method}

Let the matrix variable $X$ in the problem NSDP and consequently in the trust region problem QPTR be decomposed as $X=(F, L) \in \mathbb{R}^{p \times r} \times S^{n}$. Then, the problem QPTR can be rewritten in the form:

$$
\begin{aligned}
\min _{\Delta F, \Delta L} & q^{\sigma}(\Delta F, \Delta L) \\
\text { s.t. } & H_{F}(\cdot) \Delta F+H_{L}(\cdot) \Delta L+H(\cdot)=0, \\
& (F+\Delta F, L+\Delta L, V+\Delta V) \in \mathcal{F}_{s},\|(\Delta F, \Delta L)\| \leq \delta,
\end{aligned}
$$


where

$$
\begin{aligned}
q^{\sigma}(\Delta F, \Delta L)= & \mathcal{L}_{F}^{\sigma} \Delta F+\mathcal{L}_{L}^{\sigma} \Delta L+\frac{1}{2}\left(\mathcal{L}_{F F}^{\sigma}(\Delta F, \Delta F)\right. \\
& \left.+2 \mathcal{L}_{F L}^{\sigma}(\Delta F, \Delta L)+\mathcal{L}_{L L}^{\sigma}(\Delta L, \Delta L)\right)
\end{aligned}
$$

is the quadratic approximation of $\mathcal{L}^{\sigma}$, and $\delta$ is the trust-region radius.

In order to avoid possible infeasibility when solving (15) we use the tangent space approach (see, e.g., [4]). In this approach, the solution $\Delta X$ of the trust region problem is a decomposition of a normal step $\Delta X^{n}$ and a tangential step $\Delta X^{t}$; each one of them is obtained by solving an unconstrained trust region subproblem.

The tangent space approach relies on the null space operator of the Jacobian of the equality constraint. A drawback of the utilisation of directional derivatives is the lack of an explicit form of the Jacobian matrix. In the following Lemma we use a technique that is often used in optimal control to provide this null space operator, which does not require such a matrix explicitly.

Lemma 3.1. Let $(F, L, V) \in \mathcal{F}_{s}, \gamma>0, \Delta L \in \mathbb{R}^{n \times n}$ be given. The range space of the linear operator $\mathcal{T}(F, L)$ defined by

$$
\mathcal{T}(F, L)=\left(-H_{L}^{-1}(F, L) H_{F}(F, L), \mathcal{I}\right)
$$

coincides with the null space $\mathcal{N}\left(\nabla H^{T}(F, L)\right)$ of the Jacobian of the equality constraint, where $\mathcal{I}$ is the identity operator.

Proof. The operator $H_{L}(F, L)$ is linear (see (11)) and is also bijective (see [9, Lemma 5]), then $H_{L}(F, L)$ is invertible. Hence, the linearized equality constraint in (15) implies

$$
\Delta L=-H_{L}^{-1}(F, L) H_{F}(F, L) \Delta F-H_{L}^{-1}(F, L) H(F, L) .
$$

This leads to the following decomposition of $\Delta X=(\Delta L, \Delta F)$ :

$$
(\Delta L, \Delta F)=\mathcal{T}(F, L) \Delta F+\left(-H_{L}^{-1}(F, L) H(F, L), 0\right),
$$


where 0 is the zero operator. The null space of the Jacobian $\nabla H^{T}$ is given by

$$
\begin{aligned}
\mathcal{N}\left(\nabla H^{T}\right) & =\left\{(\Delta F, \Delta L) \in \mathbb{R}^{n_{u} \times n_{y}} \times \mathbb{R}^{n_{x} \times n_{x}} \mid H_{F, L}(F, L)(\Delta F, \Delta L)=0\right\} \\
& =\left\{\mathcal{T}(F, L) \Delta F, \quad \Delta F \in \mathbb{R}^{n_{u} \times n_{y}}\right\}=\mathcal{R}(\mathcal{T}(F, L)),
\end{aligned}
$$

where $\mathcal{R}(\mathcal{T})$ is the range space of $\mathcal{T}$.

As seen above the step $\Delta X$ solution of (15) is decomposed as (see (16)):

$$
\begin{aligned}
\Delta X & =(\Delta L, \Delta F)=\mathcal{T}(F, L) \Delta F+\left(-H_{L}^{-1}(F, L) H(F, L), 0\right) \\
& =\left(\Delta L^{t}, \Delta F\right)+\left(\Delta L^{n}, 0\right)=\Delta X^{t}+\Delta X^{n} .
\end{aligned}
$$

The following Lemma shows that the linearized equality constraint in the problem (15) is splitted into two Lyapunov equations.

Lemma 3.2. Let $(F, L, V) \in \mathcal{F}_{s}, K \in \mathbb{R}^{n \times n}$ be given, and let $(\Delta F, \Delta L) \in$ $\mathbb{R}^{n_{u} \times n_{y}} \times \mathbb{R}^{n_{x} \times n_{x}}$ be the solution of (15), and let $\gamma>0$ be a given constant. The linearized equality constraint in (15) is decomposed into the following Lyapunov equations

$$
\begin{gathered}
\Delta L^{n} \tilde{A}(\cdot)+\tilde{A}(\cdot)^{T} \Delta L^{n}+H(F, L)=0 \\
\Delta L^{t}(\Delta F) \tilde{A}(\cdot)+\tilde{A}(\cdot)^{T} \Delta L^{t}(\Delta F)+(\Delta F C)^{T} N(F, L) \\
+N(F, L)^{T} \Delta F C=0,
\end{gathered}
$$

where

$$
\begin{aligned}
& \tilde{A}(\cdot)=\left(A(F)+\frac{1}{\gamma^{2}} B_{1} B_{1}^{T} L\right), \\
& A(F)=(A+B F C), \\
& N(F, L)=\left(B^{T} L+D_{1}^{T} C(F)\right) .
\end{aligned}
$$

Proof. (See also [10, Lemma 2.2] for a similar result but on the problem NSDP2) From the step decomposition (17) the linearized equality constraint of (15) can be rewritten as

$$
H_{L}(F, L) \Delta L^{t}+H_{F}(F, L) \Delta F+H_{L}(F, L) \Delta L^{n}+H(F, L)=0 .
$$


Since $\Delta X^{t}=\mathcal{T}(F, L) \Delta F$ lies in the null space of the Jacobian $\nabla H^{T}(F, L)$, then

$$
H_{L}(F, L) \Delta L^{t}+H_{F}(F, L) \Delta F=0,
$$

which by using the derivatives (11) implies (19). Hence, the linearized equality constraint reduces to

$$
H_{L}(F, L) \Delta L^{n}+H(F, L)=0,
$$

and by using (11) gives (18).

An important feature of the tangent space approach is the decomposition of the trust region problem (15) into two unconstrained trust region subproblems. The first subproblem is:

$$
\text { (NTR) } \quad \min _{\left\|\Delta L^{n}\right\| \leq \delta}\left\|H_{L}(F, L) \Delta L^{n}+H(F, L)\right\|^{2} .
$$

It is particularly desirable to obtain an approximate solution $\Delta X^{n}=\left(\Delta L^{n}, 0\right)$ to (20). An efficient solution can be obtained, however, by solving the linear matrix equation

$$
H_{L}(F, L) \Delta L^{n}+H(F, L)=0,
$$

in $\Delta \hat{L}^{n}$ followed by rescaling $\Delta \hat{L}^{n}$ to lie within the trust region, i.e., $\Delta L^{n} \rightarrow$ $v \delta \hat{L}^{n}$, where the scaling parameter $v$ is given by

$$
v= \begin{cases}1 & \text { if }\left\|\Delta \hat{L}^{n}\right\| \leq \delta, \\ \frac{\delta}{\left\|\Delta \hat{L}^{n}\right\|} & \text { otherwise. }\end{cases}
$$

Observe that the linear matrix equation (21) is simply the Lyapunov equation (18). Roughly speaking, the computation of $\Delta X^{n}$ reduces to solving one Lyapunov equation per iteration.

Having computed $\Delta X^{n}$, the tangential step $\Delta X^{t}(\Delta F)=\left(\Delta L^{t}(\Delta F), \Delta F\right)$ is obtained as a solution of the following unconstrained trust region subproblem:

(TTR) : $\left\{\begin{array}{cl}\min _{\Delta F} & q^{\sigma}(\Delta F), \\ \text { s.t. } & \|\Delta F\| \leq \delta, \quad\left(F+\Delta F, L+\Delta L^{n}+\Delta L^{t}, V+\Delta V\right) \in \mathcal{F}_{s},\end{array}\right.$ 
where

$$
\begin{aligned}
q^{\sigma}(\Delta F)= & \mathcal{L}_{F}^{\sigma} \Delta F+\mathcal{L}_{L}^{\sigma}\left(\Delta L^{t}(\Delta F)\right)+\mathcal{L}_{F L}^{\sigma}\left(\Delta F, \Delta L^{n}\right) \\
& +\mathcal{L}_{L L}^{\sigma}\left(\Delta L^{t}(\Delta F), \Delta L^{n}\right)+\frac{1}{2}\left[\mathcal{L}_{F F}^{\sigma}(\Delta F, \Delta F)\right. \\
& \left.+2 \mathcal{L}_{F L}^{\sigma}\left(\Delta F, \Delta L^{t}(\Delta F)\right)+\mathcal{L}_{L L}^{\sigma}\left(\Delta L^{t}(\Delta F), \Delta L^{t}(\Delta F)\right)\right],
\end{aligned}
$$

and $\Delta L^{t}(\Delta F)$ solves the Lyapunov equation (19).

Applying first-order necessary optimality conditions on the subproblem TTR give the following result.

Lemma 3.3 [10, lemma 2.5]. Let $(F, L, V) \in \mathcal{F}_{s}, \sigma, \gamma>0$ be given. Assume that $\Delta F \in \mathbb{R}^{n_{u} \times n_{y}}$ is a solution of TTR, then $\Delta F$ satisfies the linear matrix equation

$$
\begin{aligned}
\mathcal{U}(\Delta F) \equiv & N(F, L)\left(\Delta K(\Delta F)+\sigma N(F, L)^{T} \Delta F C\right. \\
& +\sigma C^{T} \Delta F^{T} N(F, L)+\sigma \Delta L^{t}(\Delta F) \tilde{A}(\cdot)+\sigma \tilde{A}(\cdot)^{T} \Delta L^{t}(\Delta F) \\
& \left.+\sigma \Delta Z_{1}(\Delta F)+\frac{2}{\gamma^{2}} \Delta Z_{2}(\Delta F)\right) C^{T} \\
& +\left(B^{T} \Delta L^{t}(\Delta F)+D_{1}^{T} D_{1} \Delta F C\right)(K+\sigma H(\cdot)) C^{T}+\lambda \Delta F \\
= & -\left(B^{T} \Delta L^{n}+N(F, L)\right)(K+\sigma H(\cdot)) C^{T} \\
& \left.-N(F, L)\left(Z_{0}+\sigma \Delta K^{n}+H_{L} \Delta L^{n}\right)\right) C^{T},
\end{aligned}
$$

where $N(F, L)=\left(B^{T} L+D_{1}^{T} C(F)\right)$, and $K, \Delta L^{t}$ solve (13) (with $\sigma=0$ ), (19), respectively. Furthermore, $Z_{0}, \Delta K^{n}, \Delta L^{t}, \Delta K^{t}, \Delta Z_{1}$, and $\Delta Z_{2}$ solve the Lyapunov equations (23)-(27), respectively, and $\lambda$ is the Lagrange multiplier associated with the trust-region constraint.

$$
\begin{aligned}
Z_{0} A(F)^{T}+ & A(F) Z_{0}+B_{1} B_{1}^{T}+\tilde{A}(\cdot)(K+\sigma H(\cdot)) \\
+ & (K+\sigma H(\cdot)) \tilde{A}(\cdot)^{T}=0, \\
\Delta K^{n} A(F)^{T} & +A(F) \Delta K^{n}+\tilde{A}(\cdot) \tilde{A}(\cdot)^{T} \Delta L^{n}+\Delta L^{n} \tilde{A}(\cdot)^{T} \tilde{A}(\cdot) \\
& +(K+\sigma H(\cdot)) \Delta L^{n} B_{1} B_{1}^{T}+B_{1} B_{1}^{T} \Delta L^{n}(K+\sigma H(\cdot)) \\
& +2 \tilde{A}(\cdot) \Delta L^{n} \tilde{A}(\cdot)^{T}=0,
\end{aligned}
$$




$$
\begin{aligned}
& \Delta K^{t}(\Delta F) A(F)^{T}+A(F) \Delta K^{t}(\Delta F)+(K+\sigma H(\cdot))(B \Delta F C)^{T} \\
&+ B \Delta F C(K+\sigma H(\cdot))=0 \\
& \Delta Z_{1} A(F)^{T}+ A(F) \Delta Z_{1}+C^{T} \Delta F^{T}(N(F, L) \tilde{A}(\cdot)+\tilde{A}(\cdot) N(F, L)) \\
&+\left(\tilde{A}(\cdot) N(F, L)+N(F, L)^{T} \tilde{A}(\cdot)^{T}\right) \Delta F C \\
&+ 2 \tilde{A}(\cdot) \tilde{A}(\cdot)^{T} \Delta L^{t}(\Delta F)+2 \Delta L^{t}(\Delta F) \tilde{A}(\cdot) \tilde{A}(\cdot)^{T}=0 \\
& \Delta Z_{2} A(F)^{T}+ A(F) \Delta Z_{2}+\tilde{A}(\cdot) \Delta L^{t}(\Delta F) \tilde{A}(\cdot) \\
&+ \tilde{A}(\cdot)^{T} \Delta L^{t}(\Delta F) \tilde{A}(\cdot)^{T}=0,
\end{aligned}
$$

where

$$
\begin{aligned}
& \tilde{A}(\cdot)=\left(A(F)+\frac{1}{\gamma^{2}} B_{1} B_{1}^{T} L\right), \\
& A(F)=(A+B F C), \\
& N(F, L)=\left(B^{T} L+D_{1}^{T} C(F)\right) .
\end{aligned}
$$

Proof. By using the results of Lemma 2.1, the direct differentiation of $q^{\sigma}(\Delta F)$ with respect to $\Delta F$ yields Newton's equation (22) coupled with the aforementioned associated Lyapunov equations. The following two properties of trace derivatives have been particularly used to derive that equation

$$
\frac{\partial \operatorname{Tr}\left(M_{1} \Delta F M_{2}\right)}{\partial \Delta F}=M_{1}^{T} M_{2}^{T}, \quad \frac{\partial \operatorname{Tr}\left(M_{1} \Delta F^{T} M_{2}\right)}{\partial \Delta F}=M_{2} M_{1},
$$

where $M_{1}$ and $M_{2}$ are matrices of appropriate dimensions.

Observe that, the coupled Lyapunov equations (23)-(27) arise as a result of differentiating all those terms of $\Delta L^{t}(\Delta F)$ in the quadratic model $q^{\sigma}(\Delta F)$ with respect to $\Delta F$.

The approximate solutions of the unconstrained trust region subproblems NTR and TTR is described in the next Algorithm. The Algorithm starts by computing the normal step $\Delta X^{n}=\left(\Delta L^{n}, 0\right)$ followed by solving the linear matrix equation (22) coupled with the Lyapunov equations (19) and (23)-(27) to obtain $\Delta X^{t}(\Delta F)=\left(\Delta L^{t}(\Delta F), \Delta F\right)$. A conjugate gradient trust region method is considered to compute $\Delta X^{t}(\Delta F)$ approximately. 
Within the CG trust region method the iterates are forced to satisfy the inequality constraints (3). This means that the computed step by the CG method is enforced to fulfill the condition:

$$
\left(F+\Delta F, L+\Delta L^{n}+\Delta L^{t}(\Delta F), V+\Delta V\right) \in \mathcal{F}_{s} .
$$

If the computed trial step does not lie strictly within $\mathcal{F}_{s}$, then such a step is rejected and a decrease in $\delta$ takes place.

Remark 3.1. The problem NSDP1 can be simplified by leaving the variable $L$ to replace $V$ in both inequality constraints. This idea reduces the computations in ALSQP to two Lyapunov equations for computing $V$ and $\Delta V(\Delta F)$, and clearly increases the efficiency of the method ALSQP. In this case the computed step $\Delta X=\left(\Delta F, \Delta L^{n}+\Delta L^{t}(\Delta F)\right)$ is forced to satisfy the coupled inequality constraints in the compact form:

$$
\left(F+\Delta F, L+\Delta L^{n}+\Delta L^{t}(\Delta F)\right) \in \mathcal{F}_{s} .
$$

The following Algorithm describes the computation of the steps $\Delta X^{n}$ and $\Delta X^{t}(\Delta F)$, where $\Delta X^{n}$ is computed first followed by computing $\Delta X^{t}(\Delta F)$.

Algorithm 3.1. (Computing $\Delta X^{n}$ and $\Delta X^{t}(\Delta F)$ solutions of NTR and TTR)

I. Computing the normal step $\Delta X^{n}$

Let $(F, L) \in \mathcal{F}_{s}, K \in \mathbb{R}^{n \times n}, \sigma>0$, and the constant matrices $A, B, B_{1}$, $C, C_{1}, D_{1}$ be given.

i. Solve the Lyapunov equation (18) for $\Delta L^{n}$

ii. Scale $\Delta L^{n}$ so that $\left\|\Delta L^{n}\right\| \leq \delta$

II. Computing $\Delta X^{t}(\Delta F)$

Given $\Delta L^{n}$, solve (23)-(24), and (13) (with $\sigma=0$ ) for $Z_{0}, \Delta K^{n}$, $K \in \mathbb{R}^{n \times n}$, respectively. Let $\tilde{\epsilon} \in(0,1)$. Set $T_{0}=0_{n_{u} \times n_{y}}$, and compute the residual (the R.H.S. of (22)): 


$$
\begin{aligned}
U_{0}= & \left.-N(F, L)\left((K+\sigma H(\cdot))+Z_{0}+\sigma \Delta K^{n}+H_{L} \Delta L^{n}\right)\right) C^{T} \\
& -B^{T} \Delta L^{n}(K+\sigma H(\cdot)) C^{T} .
\end{aligned}
$$

Then, set $D_{0}=U_{0}$.

Repeat at most $n_{u} \times n_{y}$ times

1. Solve for $\Delta L^{t}(D), \Delta K^{t}(D), \Delta Z_{1}(D)$, and $\Delta Z_{2}(D)$ the Lyapunov equations (19), (25)-(27), respectively.

2. Compute the ratio $\xi=\frac{\|U\|^{2}}{\langle D, \mathcal{U}(D)\rangle}$ and then the parameter

$$
\begin{aligned}
\tilde{\tau}=\max \{ & \tau>0:\|T+\tau D\| \leq \delta, L+\Delta L^{n}+\Delta L^{t}(\tau) \succ 0, \\
& -\Delta Y(\tau) \succ 0\},
\end{aligned}
$$

where

$$
\begin{aligned}
\Delta Y(\tau)= & (A+B(F+T+\tau D) C) \Delta L^{t}(\tau) \\
& +\Delta L^{t}(\tau)(A+B(F+T+\tau D) C)^{T}
\end{aligned}
$$

3. If $\xi>\tilde{\tau}$ or $\xi \leq 0$, then set $\Delta F=T+\tilde{\tau} D$, and stop; otherwise, set $T^{+}=T+\xi D$.

4. Update the residual: $U^{+}=U-\xi \mathcal{U}(D)$, and set $\epsilon_{c g}=\min \left\{\tilde{\epsilon},\left\|U^{0}\right\|\right\}$.

5. If $\frac{\left\|U^{+}\right\|}{\left\|U^{0}\right\|} \leq \epsilon_{c g}$, set $\Delta F=T^{+}$and stop; otherwise go to the next step.

6. Compute $\zeta=\frac{\left\|U^{+}\right\|^{2}}{\|U\|^{2}}$, set $D^{+}=U^{+}+\zeta D$, and go to step 1 .

\section{End (repeat)}

Having computed the trial step $\Delta X=\left(\Delta F, \Delta L^{n}+\Delta L^{t}(\Delta F)\right)$ and the new multiplier estimate $K_{k+1}$ it remains to accept or reject this step and to increase or decrease the trust region radius $\delta_{k}$ according to the strategy of the trust region method (see [4]). The augmented Lagrangian function (2) is used as a merit function. The quantities $\operatorname{Ared}(\Delta X ; \sigma)$ and $\operatorname{Pred}(\Delta X ; \sigma)$ of the "actual" and 
"predicted" reductions of this merit function are used to measure progress made by the computed trial step towards optimality and feasibility, which are defined as

$$
\operatorname{Ared}(\Delta X ; \sigma)=\mathcal{L}^{\sigma}\left(X_{k}, K_{k}\right)-\mathcal{L}^{\sigma}\left(X_{k}+\Delta X, K_{k+1}\right),
$$

and

$$
\begin{gathered}
\operatorname{Pred}(\Delta X ; \sigma)=q_{k}(0)-q_{k}(\Delta X) \\
+\sigma\left[\left\|H\left(X_{k}\right)\right\|^{2}-\left\|H\left(X_{k}\right)+H_{L}\left(X_{k}\right) \Delta L+H_{F}\left(X_{k}\right) \Delta F\right\|^{2}\right],
\end{gathered}
$$

where $X_{k}=\left(F_{k}, L_{k}\right)$. Note that from the problem structure the last term in $\operatorname{Pred}(\Delta X ; \sigma)$ is exactly the linearized model of the equality constraint of (15). We know from Lemma 3.2 that the linearized equality constraint is decomposed into the two Lyapunov equations (18)-(19), which are solved in every iteration of the Algorithm ALSQP. As a result, $\operatorname{Pred}(\Delta X ; \sigma)$ reduces to the simpler form

$$
\operatorname{Pred}(\Delta X ; \sigma)=q_{k}(0)-q_{k}(\Delta X)+\sigma\left\|H\left(X_{k}\right)\right\|^{2} .
$$

The ratio $r_{k}=\operatorname{Ared}(\Delta X ; \sigma) / \operatorname{Pred}(\Delta X ; \sigma)$ is used to measure progress towards optimality and feasibility. According to the value of $r_{k}$ the computed trial step $\Delta X$ is accepted or rejected, and consequently $\delta_{k}$ is increased or decreased.

The constrained trust region Algorithm is stated in the following lines, which represents the major part of the Algorithm ALSQP, namely in item $c$. The update of the step and the multiplier mentioned in item $d$ of the Algorithm ALSQP are stated more specifically below in the Algorithm.

Algorithm 3.2. (The constrained trust region Algorithm)

Given $\mu_{i}, i=1,2$ with $0<\mu_{1}<\mu_{2}<1, \bar{\sigma}>0, \sigma_{0} \geq 1$, choose $\left(F_{0}, L_{0}\right) \in \mathcal{F}_{s}$, and $K_{0}$ solution of (13) (with $\sigma=0$ ). Set $k=0$.

While (5) is not satisfied, do

1. Compute $\Delta L_{k}^{n}$ and $\Delta F_{k}$ by the Algorithm 3.1. Given $\Delta F_{k}$, solve (19) to obtain $\Delta L^{t}\left(\Delta F_{k}\right)$, and then set $\Delta L_{k}=\Delta L_{k}^{n}+\Delta L^{t}\left(\Delta F_{k}\right)$.

2. Compute the new multiplier estimate $K_{k+1}$, e.g., by (13) (with $\sigma=0$ ). 
3. Compute Ared $_{k}$ and Pred $_{k}$ by using (28) and (29), respectively. If

$$
\operatorname{Pred}_{k}<\frac{\sigma_{k-1}}{2}\left\|H_{k}\right\|^{2}
$$

then set

$$
\sigma_{k}=\frac{2\left(q_{k}\left(X_{k}\right)-q_{k}(0)\right)}{\left\|H_{k}\right\|^{2}}+\bar{\sigma} ;
$$

otherwise, set $\sigma_{k}=\sigma_{k-1}$.

4. Compute the ratio $r_{k}=\operatorname{Ared}_{k} / \operatorname{Pred}_{k}$, update $\delta_{k}$, and accept or reject the step according to the following:

$$
\begin{aligned}
& \text { If } r_{k}<\mu_{1}, \\
& \quad \text { set } \delta_{k+1}=\frac{\delta_{k}}{2} \text { and reject the trial step. } \\
& \text { Else if } \mu_{1} \leq r_{k}<\mu_{2}, \\
& \quad \text { set } \delta_{k+1}=\delta_{k}, F_{k+1}=F_{k}+\Delta F_{k}, L_{k+1}=L_{k}+\Delta L_{k} . \\
& \text { Else if } r_{k} \geq \mu_{2}, \\
& \quad \text { set } \delta_{k+1}=2 \delta_{k}, F_{k+1}=F_{k}+\Delta F_{k}, L_{k+1}=L_{k}+\Delta L_{k} .
\end{aligned}
$$

End (If)

\section{End (do)}

In the computations the following values have been assigned to the parameters in the Algorithm 3.2: $\mu_{1}=0.1, \mu_{2}=0.7$, and $\bar{\sigma}=1$. We use the following initial values: $\delta_{0}=10^{2}$, and $\sigma_{0}=1$.

\section{Numerical results}

In this Section, an implementation for the Algorithm ALSQP is described. A Matlab code was written corresponding to this implementation. The constant $\gamma>0$ of the problem NSDP1 is initially estimated using the Matlab function hinflmi from the LMI Control System Toolbox. On the other hand, we need to solve several Lyapunov equations during the computation of the trial step. The Matlab function $\operatorname{lyap}(\cdot, \cdot)$ from the Control System Toolbox is used to solve approximately those equations. 
All considered test problems were chosen from the benchmark collection COMPleib [8]. Obviously, for every test problem an initial point $\left(F_{0}, L_{0}\right) \in \mathcal{F}_{S}$ is required. One successful approach is to choose an $F_{0}$ from the following set:

$$
D_{s}=\left\{F \in \mathbb{R}^{p \times r} \mid \operatorname{Re}\left(v_{i}(A(F))\right)<0, i=1, \ldots, n_{x}\right\},
$$

where $\operatorname{Re}\left(v_{i}(A(F))\right)$ are the real-parts of the eigenvalues of $A(F)$. From Lyapunov stability theory, (see, e.g., [15, Theorem 11]) there is an equivalence between the following:

1. There exists $F \in D_{s}$.

2. There exists $L \succ 0$ such that $Y(F, L) \prec 0$.

3. For every $C(F)^{T} C(F)$ there exists a unique solution $L$ of the Lyapunov equation (14), and if $C(F)^{T} C(F) \succ 0$ then $L \succ 0$.

Hence, by choosing $F_{0} \in D_{s}$ such that $C\left(F_{0}\right)^{T} C\left(F_{0}\right) \succ 0$, then the $L_{0}$ solution of the Lyapunov equation (14) is strictly feasible with respect to the inequality constraints:

$$
L_{0} \succ 0, \quad Y\left(F_{0}, L_{0}\right) \prec 0 .
$$

However, an initial $F_{0} \in D_{s}$ can be determined, e.g., by using the code slpmm [7].

The performance of the ALSQP method is compared numerically with the CTR method developed in [9], the IPCTR method proposed in [10], and Newton's method combined with an Armijo stepsize rule as proposed in [16]. In the numerical examples, we denote the Newton Algorithm by Armijo. Note that the two methods CTR and IPCTR are based on the simpler problem NSDP2. On the other hand, the problem NSDP2 was formulated in [16] as an unconstrained minimization problem in the variable $F$ and was solved by the above mentioned method.

In the following we consider two numerical examples from [8] that can be cast as nonlinear semi-definite programs of the form (1).

The ALSQP method terminates if the stopping criterion (5) reaches accuracy of $10^{-7}$. 
Example 4.1. The first test problem describes the longitudinal motion of a VTOL helicopter (see [8, HE1]). The data matrices of the continuous-time linearized state space model (see (6)) are the following:

$$
\begin{aligned}
A & =\left[\begin{array}{rrrr}
-0.0366 & 0.0271 & 0.0188 & -0.4555 \\
0.0482 & -1.0100 & 0.0024 & -4.0208 \\
0.1002 & 0.3681 & -0.7070 & 1.4200 \\
0 & 0 & 1 & 0
\end{array}\right], \\
B & =\left[\begin{array}{rr}
0.4422 & 0.1761 \\
3.5446 & -7.5922 \\
-5.5200 & 4.4900 \\
0 & 0
\end{array}\right], \quad B 1=\left[\begin{array}{rr}
0.0468 & 0 \\
0.0457 & 0.0099 \\
0.0437 & 0.0011 \\
-0.0217 & 0
\end{array}\right], \\
C^{T} & =\left[\begin{array}{l}
0 \\
1 \\
0 \\
0
\end{array}\right], \quad C 1^{T}=\left[\begin{array}{rr}
\sqrt{2} & 0 \\
0 & \frac{1}{\sqrt{2}} \\
0 & 0 \\
0 & 0
\end{array}\right], \quad D_{1}=\frac{1}{\sqrt{2}}\left[\begin{array}{ll}
1 & 0 \\
0 & 1
\end{array}\right],
\end{aligned}
$$

The main goal is to compute an optimal SOF gain matrix $F_{*}$ that meets a desired performance criterion, and at the same time the computed $F_{*}$ must stabilize (in the Lyapunov sense) the closed loop control system (8). Equivalent to this task is to solve the optimization problem NSDP1 for finding a stationary point $\left(F_{*}, L_{*}\right)$.

The zero matrix $F_{0}=0_{n_{u} \times n_{y}}$ is such that $\left(F_{0}, L_{0}\right) \notin \mathcal{F}_{s}$. Therefore, the following point $\left(F_{0}, L_{0}\right)$ is considered:

$$
F_{0}=\left[\begin{array}{r}
-0.7674 \\
1.4125
\end{array}\right],
$$

and $L_{0}$ is the corresponding solution of the Lyapunov equation (14).

In Table 1 the convergence rate for the method ALSQP is shown. The computed static output feedback gain matrix is

$$
F_{*}=\left[\begin{array}{r}
-1.6277 \\
6.5100
\end{array}\right] \text {. }
$$

Table 2 gives a comparison between ALSQP and the other solvers on this problem for the same starting point $\left(F_{0}, L_{0}\right)$. These results show that ALSQP is 


\begin{tabular}{c|c|c|c|c}
\hline$k$ & $J_{k}$ & $\left\|\nabla \mathcal{L}_{F_{k}}^{\sigma}\right\|+\left\|H_{k}\right\|$ & $i_{c g}$ & $\sigma_{k}$ \\
\hline 0 & $1.5061 \mathrm{e}+001$ & $1.313 \mathrm{e}+000$ & - & 1 \\
1 & $1.3683 \mathrm{e}+001$ & $1.916 \mathrm{e}+000$ & 1 & 1 \\
2 & $1.3508 \mathrm{e}+001$ & $2.304 \mathrm{e}+000$ & 2 & 1 \\
3 & $1.3314 \mathrm{e}+001$ & $1.245 \mathrm{e}+000$ & 2 & 1 \\
4 & $1.3296 \mathrm{e}+001$ & $1.339 \mathrm{e}-001$ & 2 & 1 \\
5 & $1.3306 \mathrm{e}+001$ & $1.442 \mathrm{e}-001$ & 2 & 1 \\
6 & $1.3311 \mathrm{e}+001$ & $4.787 \mathrm{e}-003$ & 2 & 1 \\
7 & $1.3311 \mathrm{e}+001$ & $5.936 \mathrm{e}-006$ & 2 & 1 \\
8 & $1.3311 \mathrm{e}+001$ & $4.535 \mathrm{e}-012$ & 2 & 1 \\
\hline
\end{tabular}

Table 1 - Performance of ALSQP on Example 4.1.

\begin{tabular}{l|c|c|c|c}
\hline Method & Armijo & CTR & IPCTR & ALSQP \\
\hline No. of iterations & 10 & 19 & $15(4)$ & 8 \\
\hline
\end{tabular}

Table 2 - Comparison between the four methods on Example 4.1.

quite competitive with other solvers on this problem with respect to the number of iterations.

Figure 1 shows the effect of the computed SOF gain $F_{*}$ on the closed-loop control system (8). In particular, $F_{*}$ enforces all state variables to converge to zero.

Example 4.2. The second test problem is the Tenyain following model (see [8, $\mathrm{TF} 1])$. The given data matrices for this example are as follows:

$$
A=\left[\begin{array}{rrrrrrr}
-1 & 0 & 0 & 0 & 0 & 0 & 0 \\
1 & 0 & 0 & 0 & 0 & 0 & 0 \\
0 & 1 & 0 & 0 & 0 & 0 & 0 \\
0 & 0 & 0 & 0 & 0 & 0 & 0 \\
0 & 0 & 0 & 1 & -1 & 0 & 0 \\
-0.088 & 0.0345 & 0 & 0 & 1 & -0.0032 & 0 \\
0 & 0 & 0.05 & 0 & 0 & 0 & -1 e-5
\end{array}\right],
$$



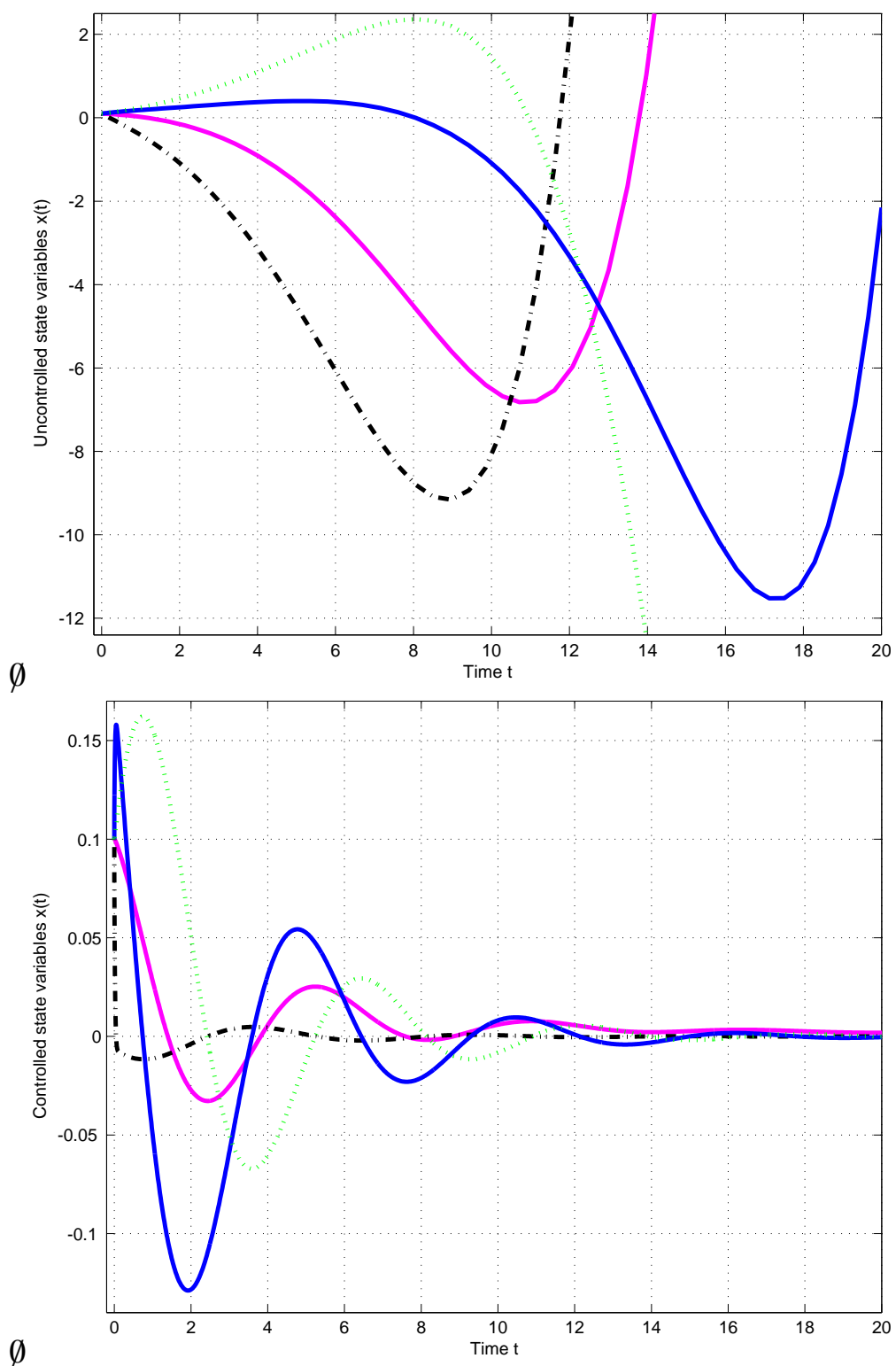

Figure 1 - Uncontrolled and controlled state space models for Example 4.1. 


$$
\begin{aligned}
& B=\left[\begin{array}{rr}
1 & 0 \\
0 & 0 \\
0 & 0 \\
0 & 0.09 \\
0 & 0 \\
0 & 0
\end{array}\right], B 1=\left[\begin{array}{r}
0_{6 \times 1} \\
-0.05
\end{array}\right], \quad C=\left[\begin{array}{lllllll}
0 & 0 & 1 & 0 & 0 & 0 & 0 \\
0 & 1 & 0 & 0 & 0 & 0 & 0 \\
0 & 0 & 0 & 0 & 0 & 0 & 1 \\
0 & 0 & 0 & 0 & 0 & 1 & 0
\end{array}\right], \\
& {\left[\begin{array}{ll}
0 & 0
\end{array}\right]} \\
& C_{1}=\left[\begin{array}{rrr}
0 & 1 \\
04 \times 5 & 2.23 & 0 \\
& 0 & 0 \\
0 & 0
\end{array}\right], D_{1}=\left[\begin{array}{rr}
0 & 0 \\
0 & 0 \\
1.7321 & 0 \\
0 & 0.5477
\end{array}\right] \text {. }
\end{aligned}
$$

The zero matrix $F_{0}=0_{n_{u} \times n_{y}}$ implies that $\left(F_{0}, L_{0}\right)=\left(0_{n_{u} \times n_{y}}, L_{0}\right) \notin \mathcal{F}_{s}$, where $L_{0}$ is the corresponding solution of the Lyapunov equation (14). Therefore, the following $F_{0}$ is chosen:

$$
F_{0}=\left[\begin{array}{rrrr}
-0.9497 & -1.6632 & -0.1110 & 0.5383 \\
4.2917 & 2.0803 & 0.6584 & -3.5890
\end{array}\right] .
$$

Table 3 shows the performance of the method ALSQP on this example. The computed SOF gain matrix is

$$
F_{*}=\left[\begin{array}{rrrr}
-1.1897 & -2.1666 & -0.7124 & 0.8345 \\
2.3381 & -0.2038 & 1.2956 & -2.1768
\end{array}\right] .
$$

Table 4 gives a comparison between ALSQP and other solvers on this problem starting from the same point $\left(F_{0}, L_{0}\right)$.

Figure 2 shows the effect of the computed SOF feedback gain matrix $F_{*}$ on the closed-loop control system (8).

The two examples show the fast local rate of convergence of the method ALSQP starting from remote points.

In Table 5 some preliminary tests are given. For each example, we report the problem name together with the problem dimensions $\left(n_{x}, n_{u}, n_{y}, n_{w}, n_{z}\right)$, and the overall number of iterations. A dash indicates that the corresponding method 

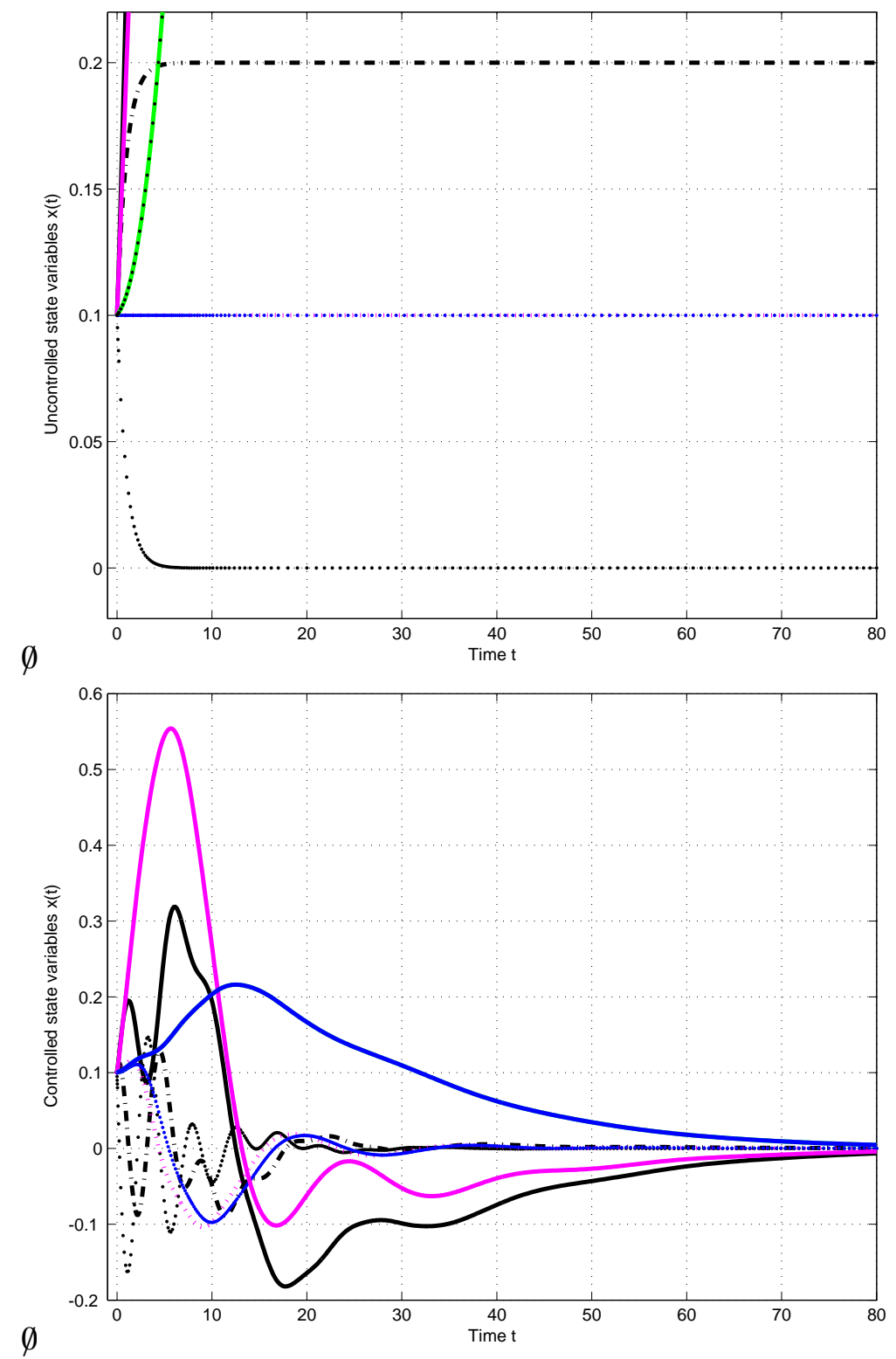

Figure 2 - Uncontrolled and controlled state space models for Example 4.2. 


\begin{tabular}{c|c|c|c|c}
\hline$k$ & $J_{k}$ & $\left\|\nabla \mathcal{L}_{F_{k}}^{\sigma}\right\|+\left\|H_{k}\right\|$ & $i_{c g}$ & $\sigma_{k}$ \\
\hline 0 & $7.6072 \mathrm{e}+002$ & $1.242 \mathrm{e}+004$ & - & 1 \\
1 & $5.9498 \mathrm{e}+002$ & $3.994 \mathrm{e}+003$ & 1 & 1 \\
2 & $5.1281 \mathrm{e}+002$ & $1.671 \mathrm{e}+003$ & 1 & 1 \\
3 & $4.3950 \mathrm{e}+002$ & $1.447 \mathrm{e}+003$ & 2 & 1 \\
4 & $3.6599 \mathrm{e}+002$ & $4.220 \mathrm{e}+002$ & 2 & 1 \\
$\vdots$ & $\vdots$ & $\vdots$ & $\vdots$ & $\vdots$ \\
12 & $2.9670 \mathrm{e}+002$ & $1.243 \mathrm{e}-001$ & 6 & 1 \\
13 & $2.9675 \mathrm{e}+002$ & $1.275 \mathrm{e}-002$ & 6 & $1.74 \mathrm{e}+000$ \\
14 & $2.9675 \mathrm{e}+002$ & $8.174 \mathrm{e}-005$ & 8 & $1.74 \mathrm{e}+000$ \\
15 & $2.9675 \mathrm{e}+002$ & $3.542 \mathrm{e}-011$ & 8 & $1.74 \mathrm{e}+000$ \\
\hline
\end{tabular}

Table 3 - Performance of ALSQP on Example 4.2.

\begin{tabular}{l|c|c|c|c}
\hline Method & Armijo & CTR & IPCTR & ALSQP \\
\hline No. of Iterations & 17 & 17 & $27(4)$ & 15 \\
\hline
\end{tabular}

Table 4 - Comparison between the four methods on Example 4.2.

fails to find an approximate solution of the considered nonconvex NSDP problems with accuracy $\epsilon_{t o l}=10^{-7}$. In particular, ALSQP was tested by using the test problem [8, REA1] from two different starting points; the results corresponding to the second starting point are denoted by REA $1 *$. In all these problems the four codes approached the same solution point.

The main conclusion that we can draw from the above results is that the method ALSQP outperforms other methods on most of the considered nonconvex and nonlinear semi-definite programming test problems.

Acknowledgements. The author wish to thank Dr. F. Leibfritz for constructive discussions on earlier version of this paper. He also would like to thank the Editor-in-Chief and an anonymous referee for their helpful comments which have improved the presentation of this paper. 


\begin{tabular}{|l|rrrrr|rrrr|}
\hline & \multicolumn{3}{|c|}{ Problem dimension } & \multicolumn{4}{|c|}{ No. of iterations } \\
\hline Name & $n_{x}$ & $n_{u}$ & $n_{y}$ & $n_{w}$ & $n_{z}$ & Armijo & CTR & IPCTR & ALSQP \\
\hline AC3 & 5 & 2 & 4 & 5 & 5 & 18 & - & $121(3)$ & 17 \\
AC6 & 4 & 2 & 2 & 7 & 7 & 9 & 9 & $21(3)$ & 9 \\
AC8 & 9 & 1 & 5 & 10 & 2 & 10 & 14 & $29(3)$ & 9 \\
AC9 & 10 & 4 & 5 & 10 & 2 & - & 15 & $36(4)$ & 14 \\
AC11 & 5 & 2 & 4 & 5 & 5 & 18 & - & $22(3)$ & 11 \\
AC15 & 4 & 2 & 3 & 4 & 6 & - & 31 & - & 28 \\
AC17 & 4 & 1 & 2 & 4 & 4 & 12 & 16 & $192(3)$ & 11 \\
DIS3 & 6 & 4 & 4 & 6 & 6 & 15 & 17 & $16(4)$ & 15 \\
HE1 & 4 & 2 & 1 & 2 & 2 & 10 & 19 & $15(4)$ & 8 \\
HE2 & 4 & 2 & 2 & 4 & 4 & 15 & 12 & $22(3)$ & 16 \\
HF1 & 130 & 1 & 2 & 1 & 2 & 7 & 12 & $38(4)$ & 6 \\
PSM & 7 & 2 & 3 & 2 & 5 & 10 & 12 & $12(4)$ & 9 \\
REA1 & 4 & 2 & 2 & 4 & 4 & 9 & 9 & $9(4)$ & 8 \\
REA1 & 4 & 2 & 2 & 4 & 4 & 19 & 16 & $21(3)$ & 16 \\
TF1 & 7 & 2 & 4 & 1 & 4 & 17 & 17 & $27(4)$ & 15 \\
TG1 & 10 & 2 & 2 & 10 & 10 & 8 & 10 & $16(4)$ & 7 \\
TMD & 6 & 2 & 4 & 1 & 3 & 10 & 8 & $9(3)$ & 8 \\
UWV & 8 & 2 & 2 & 2 & 1 & 12 & 17 & $19(3)$ & 10 \\
WEC1 & 10 & 3 & 4 & 10 & 10 & 14 & - & $19(4)$ & 11 \\
NN4 & 4 & 2 & 3 & 4 & 4 & 5 & 4 & $7(3)$ & 4 \\
NN13 & 6 & 2 & 2 & 3 & 3 & 10 & 15 & $12(3)$ & 9 \\
\hline
\end{tabular}

Table 5 - Performance of ALSQP vs. Armijo, CTR and IPCTR on test problems from [8].

\section{REFERENCES}

[1] P. Apkarian and H.D. Tuan, Concave programming in control theory, J. Global Optimization, 15 (1999), 343-370.

[2] D.S. Bernstein and W.M. Haddad, LQG control with an $\mathcal{H}_{\infty}$ performance bound: A Riccati equation approach, IEEE Transactions on Automatic Control, 34 (1989), 293-305.

[3] S.P. Boyd, L. El-Ghaoui, E. Feron and V. Balakrishnan, Linear Matrix Inequalities in System and Control Theory, Vol. 15 of SIAM Studies in Applied Mathematics, SIAM, Philadelphia, 1994. 
[4] A.R. Conn, N.I.M. Gould and Ph.L. Toint, Trust region methods, SIAM Philadelphia, in the MPS/SIAM Series on Optimization, 2000.

[5] P.P. Khargonekar and M.A. Rotea, Mixed $\mathcal{H}_{2} / \mathcal{H}_{\infty}$ control: A convex optimization approach, IEEE Transactions on Automatic Control, 36 (1991), 824-837.

[6] M. Kočvara and M. Stingl, PENNON: a code for convex nonlinear and semi-definite programming, Optimization Methods and Software, 18 (2003), 317-333.

[7] F. Leibfritz, A LMI-based algorithm for designing suboptimal static $\mathcal{H}_{2} / \mathcal{H}_{\infty}$ outputfeedback controllers, SIAM Journal on Control and Optimization, 39 (2001), 1711-1735.

[8] F. Leibfritz, COMPleib: Constraint Matrix-optimization Problem library - a collection of test examples for nonlinear semi-definite programs, control system design and related problems. Tech. Report-03, 2003. http://www.uni-trier.de/leibfr/COMPleib

[9] F. Leibfritz and E.M.E. Mostafa, Trust region methods for solving the optimal output feedback design problem, Int. J. on Control, 76 (2003), 501-519.

[10] F. Leibfritz and E.M.E. Mostafa, An interior-point trust region method for a special class of nonlinear SDP-problems, SIAM J. Optim., 12 (2002), 1048-1074.

[11] E.M.E. Mostafa, Efficient trust region methods in numerical optimization, Ph.D. thesis, Dept. of Mathematics, Faculty of Science, Alexandria University, Egypt, 2000.

[12] E.M.E. Mostafa, A trust region method for solving the decentralized static output feedback design problem, J. Appl. Math. \& Computing., 18 (2005), 1-23.

[13] T. Rautert and E.W. Sachs, Computational design of optimal output feedback controllers, SIAM J. Optim., 7 (1997), 837-852.

[14] M.A. Rotea, The generalized $\mathcal{H}_{2}$ control problem, Automatica, 29 (1993), 373-385.

[15] E.D. Sonntag, Mathematical control theory, Springer Verlag, New York, Berlin, 1990.

[16] H.T. Toivonen, A globally convergent algorithm for the optimal constant output feedback problem, Int. J. Control, 41 (1985), 1589-1599. 